\title{
RHEUMATOLOGY
}

\section{How painful is juvenile chronic arthritis?}

\author{
W Kuis, C J Heijnen, J A Hogeweg, G Sinnema, P J M Helders
}

This is the seventh article in a series on rheumatology.
Pain is an unpleasant sensory and emotional experience associated with actual or potential tissue damage ${ }^{1}$ and it is one of the major symptoms in arthritis. ${ }^{2-4}$ Pain is also the sensory modality that alerts patients so that it leads to protective responses. Pain also informs the patient that there is a potential noxious threat, which brings him or her to the doctor for diagnosis and treatment of his illness (or ailment). Unfortunately, a warning signal like acute pain can turn into chronic pain and in this stage pain itself can be the most dominant symptom and threat for the quality of life of the patient. In the pathogenesis of inflammatory pain, receptors sensitive for noxious stimuli generated as a consequence of inflammation, have a role. These receptors, called nociceptors, activate predominantly unmyelinated, small diameter sensory nerves, mediating nociceptive information to higher order neurones in the dorsal horn of the spinal medulla. On spinal as well as supraspinal level, the nociceptive signal is modulated by regulating pathways (fig 1). Besides these regulating pathways there are many substances that are able to stimulate directly and/or sensitise primary afferent nociceptive fibres (table 1$).^{56}$

Children express pain in a different way than adults do. ${ }^{7}$ This is often interpreted as if children feel less or even no pain as in the case of neonates. The idea that newborns and children differ fundamentally from adults in the perception of pain, has led to approaches that are not humane. In a survey of British paediatric anaesthetists in 1985 it appeared that $48 \%$ of the respondents never provided postoperative analgesia of any kind to neonates during or after major surgery. ${ }^{89}$

Differences in pain perception explain why there are so many discrepancies in the literature with respect to pain in children with juvenile chronic arthritis. Some investigations report that children with juvenile chronic arthritis experience substantially less pain or even no pain in comparison to adults with rheumatoid arthritis. ${ }^{10}$ However, we and others have found that children with juvenile chronic arthritis do express pain. ${ }^{11-16}$ By using algometry, we found an enhanced sensitivity in children with juvenile chronic arthritis to noxious stimulation in comparison to healthy age related controls. ${ }^{15}$ When juvenile chronic arthritis patients had actively inflamed joints, the pain threshold was reduced by $31 \%$. There was a significant inverse correlation between indices of disease activity and the pain threshold. We studied the relationship between pain threshold and pain expression as measured with visual analogue scales in a group of 57 children with juvenile chronic arthritis. By correlating three articular disease activity indices (active joint count, swollen joint count, and Ritchie index), visual analogue scales, and the functional child health assessment questionnaire with the mean pain threshold, we found that all the above mentioned items correlated negatively with the pain threshold. The latter may be interpreted that the pain threshold is related to disease activity and pain experience. No correlation was found between the mean pain threshold and the $\mathrm{C}$ reactive protein.

Huskisson and Hart found a similar correlation between the Ritchie index and pain threshold in adults with rheumatoid arthritis. ${ }^{17}$ In contrast, Walco et al found no correlation between the pain threshold and the visual analogue scales in children with juvenile chronic arthritis. ${ }^{18}$ This difference can be partly explained by the choice of pressure sites and/or by patient selection. We found a significant inverse correlation between the pain threshold and pain experience measured with visual analogue scales, which could explain $25 \%$ of the variance.$^{15}$ However, the pain threshold and the visual analogue scales measure different aspects of pain. The pain threshold as measured by the algometer might be easier to express and may be closely connected to the discharge of nociceptive afferents. In juvenile chronic arthritis the pain threshold is probably decreased as a result of the existing tissue damage in the inflamed joints. In comparison, the pain score measured with visual analogue scales is more influenced by the emotional experience associated with the disease.

An interesting phenomenon is that in juvenile chronic arthritis patients we measured a lower pain threshold of the involved joint as well as of the non-inflamed joints. ${ }^{15}$ In children with juvenile chronic arthritis in remission, we also found a lower pain threshold on all sites of measurement, although the effect was less pronounced than in children with active disease. In other words, the change in the pain threshold persists even after the input of the injured site has subsided. This shows that sensory induced changes and the impact they have on the central memory can be long lasting. ${ }^{5}$ The neurones on which these sensory induced changes can have such a longlasting impact are dorsal 


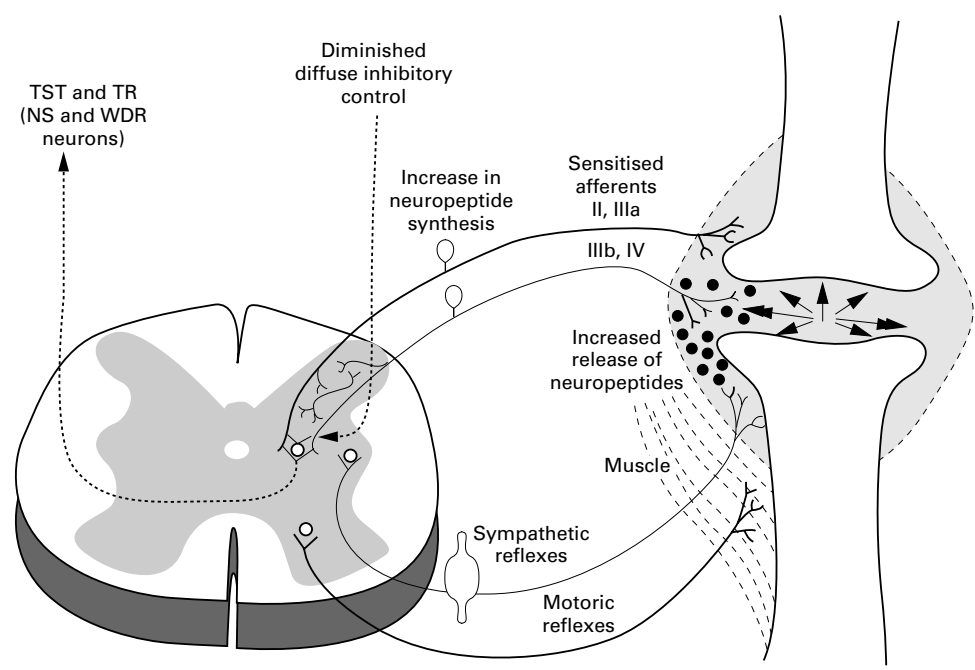

Figure 1 Neuronal events when inflammation in a joint is present. TST $=$ tractus spinothalamicus; $T S R=$ tractus spinoreticularis; $N S=$ nocispecific neurones; $W D R=$ wide dynamic range neurones; II, IIIa = myelinated afferents type II and IIIa; IIIb, IV = thinly or unmyelinated nociceptive afferents type IIIb and IV.

horn neurons and other high order projection neurons, local spinal interneurons, and long descending neurons. These longlasting structural and functional changes are often called 'neuroplastic alterations'. ${ }^{19}$ These long term facilitations are due to changes in gene regulation and/or synapse reorganisation and are part of a mechanism called 'central sensitisation'. ${ }^{20}$ Another clinical example is the phantom pain experienced by amputees, which is similar to the pain felt in the limb before amputation.

There is also experimental evidence for central sensitisation whereby a phenomenon like wind up or expansion of receptive fields of central nervous system neurons are studied. ${ }^{21}$ Besides central sensitisation there is another mechanism called peripheral sensitisation. Different local factors can be responsible for the changes at the site of the inflamed joint and can contribute to the peripheral sensitisation in arthritis, such as:

(1) Inflammation of the joint is accompanied by synovial effusion and tissue oedema. Together with the increased blood flow this may lead to an increase of the intra-articular pressure. The increased intra-articular pressure triggers the receptive endings of nociceptive fibres. The increased intra-articular temperature may increase neural velocity. ${ }^{22-24}$

(2) More nociceptors are involved during inflammation. Silent, nociceptive fibres, unresponsive before inflammation, are now receptive for various stimuli. ${ }^{25}$

(3) Many locally produced substances can stimulate directly (for example bradykinin, serotonin) and/or sensitise (for example prostaglandin PGE1, PGE2) group III and IV afferents. ${ }^{5}$ The most studied neurotransmitter of nocicep-

Table 1 Stimulating and sensitising substances for primary afferent nociceptive fibres

Stimulating substances induce, via receptor binding, depolarisation of the neuron and the formation of action potentials: for example bradykinin, histamine, serotonin, and prostaglandins

Sensitising substances lower the threshold of nociceptors, such that they become excited by non-noxious stimuli: for example 5-hydroxytryptamine, prostaglandins, bradykinin,

adenosine, serotonin, noradrenalin, leucotrienes, tachykinins, and many inflammatory mediators (interleukin-1, interleukin-6, tumour necrosis factor- $\alpha$ ) tive afferents is substance $\mathrm{P}$, which can transmit the signal to the central level (dromic direction).$^{26}$ Interestingly, substance $\mathrm{P}$ can also be directed to the peripheral ending (antidromic direction) and be released in the joint. ${ }^{27}$ Substance $\mathrm{P}$ is proinflammatory, which may contribute to the maintenance of a vicious circle of pain, noxious stimulation, and inflammation. This process is often called 'neurogenic inflammation'. ${ }^{28}$ Also in juvenile chronic arthritis peripheral sensitisation as well as central sensitisation plays a part. Evidence for central sensitisation is the general decrease in the pain threshold of non-inflamed sites of patients with active disease or in children with juvenile chronic arthritis in remission. ${ }^{15}$ We also found segmental reductions of the pain threshold ${ }^{16}$; however, the overall threshold reduction is far more pronounced than the segmental reduction.

The clinical outcome is tenderness on pressure as well as pain during movement. It is our experience that pain is more dominant in children with severe polyarticular and systemic juvenile chronic arthritis than in the oligoarticular form of the disease. This is in agreement with our finding that the mean pain threshold is correlated with the total amount of inflamed joints.

\section{Treatment}

Non-steroidal anti-inflammatory drugs inhibit PGH2 synthetase enzymes (cyclo-oxygenase) and therefore diminish the formation of stimulatory (PGE2, PGE1), and or sensitising substances (PGE2, PGE1, PGF-2 $\alpha$, PGI2) from arachidonic acid. Corticosteroids also interfere with the production of the above mentioned substances, while high doses of corticosteroids also inhibit interleukin-1 and tumour necrosis factor- $\alpha$ synthesis. ${ }^{5}$ There is evidence that intramuscular gold is neurotoxic for type IV fibres. ${ }^{29}$ So, first and second line drugs have an anti-inflammatory as well as a direct analgesic effect.

Another way to control pain is treatment directed towards controlling the nociceptive inputs in order to avoid long term sensitisation by procedures such as cooling of the inflamed joint, rest (limited movement/joint splinting), and by activation of the central nervous system. There are two relevant types of afferent mediated inhibition of nociception by peripheral stimulation of afferents. Non-nociceptive mechanical stimuli applied to sites in the same segment as the inflamed joint inhibit presynaptic primary nociceptive afferents as well as postsynaptic activation of secondary neurons in ascending tracts, involved in nociceptive processing (fig 2). There are indications that the effects of afferent stimulation in patients with chronic pain last much longer than in healthy controls. ${ }^{50}$ An example of a nonnociceptive mechanical stimulus is transcutaneous electrical nerve stimulation.

The other type of afferent mediated inhibition of nociception is known as 'counterirritation'. Small diameter (III and IV) afferents at some distance of the inflamed joint are stimulated and this results in inhibition of both small and large diameter input to the convergent wide dynamic range nerves. ${ }^{31}{ }^{32}$ An example of 


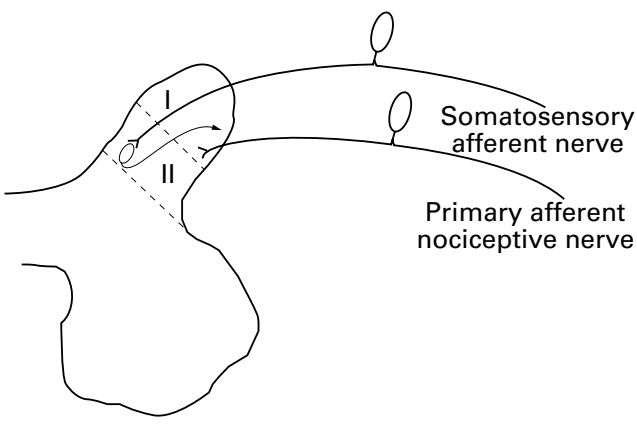

Figure 2 Non-nociceptive stimuli applied to sites on the same segment as the inflamed joint stimulate somatosensory afferent nerves. Somatosensory nerves activate inhibitory local interneurons in the substantia gelatinosa (lamina II), causing presynaptic inhibition of the primary afferent nociceptive nerves.

a treatment based on counterirritation is heat/ cold therapy. The effect of both types of afferent mediated inhibition has still to be evaluated. It is known that the placebo effect of these treatments may contribute to the effect.

Another way to control pain is to influence the central sensitisation. In children there is a growing interest in cognitive behavioural approaches, although its place in the treatment of chronic pain is still unknown. In juvenile chronic arthritis relaxation and cognitive behavioural management of pain sensitisation have proved to be moderately effective. ${ }^{33}$ Although much more research has to be done, it is questionable whether patients with a longlasting pain behaviour will benefit only from cognitive behavioural approaches. ${ }^{35}$ In addition, it may be worthwhile to consider to treat the patient with a therapy directed at the interference with central as well a peripheral sensitisation at the same time.

Moreover, it has to be emphasised that a proper and individual balance of movement versus rest is very important. Too much exercise may exacerbate symptoms and increase destruction of the joint, while movement is important for functional restoration. The documentation of peripheral and central sensitisation could help the doctor/physical therapist to dose the exercise. When the spread of sensitisation is high (low pain thresholds), mild non-painful forms of treatment appear to be the most appropriate approach. More demanding forms of treatment can be asked of the patient when a lower level of sensitisation is present. Optimal treatment should be based on a multidimensional biopsychosocial approach in a multidisciplinary team $^{36}$ in order to ensure integration of the treatment goals into the child's daily routine.

In summary there is still much debate about the role of pain in children with juvenile chronic arthritis. We have shown that pain plays an important part in the symptomatology of this condition. Better knowledge of the neurophysiology of chronic pains leads to new therapeutic regimens. Antinociceptive intervention in juvenile chronic arthritis not only serves to diminish pain, but may become increasingly important to modulate the degree of inflammation.

1 International Association for the Study of Pain. Pain supplement 3. Classification of chronic pain, descriptions of chronic pain syndromes and definitions of pain terms. Amsterdam: Elsevier Science Publishers, 1986.
2 Lovell DJ, Walco GA. Pain associated with juvenile rheumatoid arthritis. Pediatr Clin North Am 1989;36:1015-27.

3 Fitzgerald M. Arthritis and the nervous system. Trends Neurosci 1989;12:86-7.

4 Bellamy N, Bradley LA. Workshop on chronic pain, pain control and patient outcomes in rheumatoid arthritis and osteoarthritis. Arthritis Rheum 1996;3:357-62.

5 Konttinen YT, Kemppinen P, Segerberg M, et al. Peripheral and spinal neural mechanisms in arthritis, with particular reference to treatment of inflammation and pain. Arthritis Rheum 1994;7:965-82.

6 Livingstone WK. Pain mechanisms: a physiological interpretation of causalgia and its related states. New York: Plenum Press, 1976. (Reprint of the 1943 edition published by Macmillan.)

7 Owens ME. Pain in infancy: conceptual and methodological issues. Pain 1984;20:213-30.

8 Booker PD. Postoperative analgesia for neonates [Editorial]. Anaesthesia 1987;42:343-5.

9 Purcell-Jones G, Dormon F, Summer E. Paediatric anaesthesists perceptions of neonatal and infant pain. Pain 1988;33:181-7.

10 Scott PJ, Ansell BM, Huskisson EC. Measurement of pain in juvenile chronic poly arthritis. Ann Rheum Dis 1977;36: 186-7.

11 Thompson KL, Varni JW, Hanson V. Comprehensive assessment of pain in juvenile rheumatoid arthritis: an empirical model. 7 Pediatr Psychol 1987;12:241-55.

12 Walco GA, Varni JW, Illowite NT. Cognitive-behavioral pain management in children with juvenile rheumatoid arthritis. Pediatrics 1992;89:1075-9.

13 Varni JW. Evaluation and management of pain in children with juvenile rheumatoid arthritis. F Rheumatol Suppl 1992; 33:32-.5

14 Truckenbrodt H. Pain in juvenile chronic arthritis; consequences for the musculo-skeletal system. Clin Exp Rheumatol 1993;11(suppl 9): S59-S63.

15 Hogeweg JA, Kuis W, Huygen ACJ, et al. The pain threshold in juvenile chronic arthritis. Br $\mathcal{F}$ Rheumatol 1995;34:61-7.

16 Hogeweg JA, Kuis W, Oostendorp RAB, Helders PJM. General and segmental reduced pain thresholds in juvenile chronic arthritis. Pain 1995;62:11-7.

17 Huskisson EC, Hart DC. Pain threshold and arthritis. BMF 1972;iv: 193-5.

18 Walco GA, Dampier CD, Hartstein G, Djordjevic D, Miller $\mathrm{L}$. The relationship between recurrent clinical pain and pain threshold in children. In: Tyler DC, Krane EJ, eds. Advances in pain research therapy. Volume 15 pediatric pain. New York: Raven Press, 1990: 333-40.

19 Coderre JC, Katz J, Vaccarino AL, Melzack R. Contribution of central neuroplasticity to pathological pain: review of clinical and experimental evidence. Pain 1993;52:259-85.

20 Menétrey D, Besson IM. Electrophysiological characteristics of dorsal horn cells in rats with cutaneous inflammation resulting from chronic arthritis. Pain 1982;13:343-64. Woolf CJ. Generation of acute pain: central mechanisms. Br Med Bull 1991;47:523-33.

22 Stevens CR, Williams RB, Farell AJ, Blake DR. Hypoxia and inflammatory synovitis: observations and speculations. Ann Rheum Dis 1991;50:124-32.

23 Schaible HG, Schmidt RF. Effects of an experimental arthritis on the sensory properties of fine articular afferent units. I Neurophysiol 1985;54:1109-22.

24 Schaible HG, Grubb DB. Afferent and spinal mechanisms of joint pain. Pain 1993;55:5-45.

25 Hanesch U, Heppelmann B, Messlinger K, Schmidt RF. Nociception in normal and arthritis joints, structural and functional aspects. In: Willis WD, ed. Hyperalgesia and allodynia. New York: Raven Press, 1992: 81-106.

26 Kanazawa MJ, Mroz EA, Kizere M, Palkovitz M, Lee SE. Regional distribution of substance $P$ in the brain of the rat. Brain Res 1976;116:299-305.

27 Levine JD, Clark R, Devor M, Helms C, Moskowitz MA, Basbaum AI. Intraneuronal substance P contributes to the Basbaum AI. Intraneuronal substance P contributes to the

28 Levine JD, Collier DH, Basbaum AI, Moskowitz MA, Helms CA. Hypothesis: the nervous system may contribute to the pathophysiology of rheumatoid arthritis. F Rheumatol 1985;12:406-11.

29 Levine JD, Goldstine J, Mayes M, Moskowitz MA, Basbaum AI. The neurotoxic effect of gold sodium thiomalate on the peripheral nerves of the rat. Insights into the antiinflammatory actions of gold therapy. Arthritis Rheum 1986;29:897901.

30 Besson JM, Chaouch A. Peripheral and spinal mechanisms of nociception. Physiol Rev 1987;67:67-186.

31 Bouhassira D, Villaneuva L, Bing Z, LeBars D. Involvement of the subnucleus reticularis dorsalis in diffuse noxious inhibitory controls in the rat. Brain Res 1992;595:353-7.

32 Zusman M. The meaning of mechanically produced responses. Australian Physiotherapy 1994;40:35-9.

33 Walco GA, Varni JW, Ilowite NT. Cognitive-behavioral pain management in children with juvenile rheumatoid arthritis. Pediatrics 1992;89:1075-9.

34 Ross CK, Berry SL, Hayford JR, Pachman LM. Evaluation of a psychological treatment package for treating pain in uvenile rheumatoid arthritis. Arthritis Care and Research 1992;5:101-10.

35 Justins DM. Management strategies for chronic pain. Ann Rheum Dis 1996;55:588-96.

36 Vandvik IH, Hoyeraal HM. Juvenile chronic arthritis: a biobehavioural disease. Some unsolved questions. Clin Exp Rheumatol 1993;1:669-80. 